\title{
Electrical Properties of Electrorefined Nanostructured Lead (Pb) for Enhancing Radiation Safety
}

\author{
El-Sayed M. El-Refaie ${ }^{1}$, El-Gamel Amal A. ${ }^{2}$, A. H. Gepreel Mohamed ${ }^{3}$, Kandil M. ${ }^{2}$, Hussein A. Z. ${ }^{2}$ \\ ${ }^{1}$ Department Environmental Engineering, Helwan University, Faculty of Engineering, Cairo, Egypt \\ ${ }^{2}$ Department of Radiation Safety, Nuclear\&Radiological Regulatory Authority (ENRRA), Cairo, Egypt \\ ${ }^{3}$ Department of Materials Science and Engineering, Egypt-Japan University of Science and Technology (E- JUST), Alexandria, Egypt
}

Email address:

drelsaymr@yahoo.com (El-Sayed M. El-Refaie), amal.gamel@yahoo.com (El-Gamel A. A.), geprell@yahoo.com (A. H. Gepreel Mohamed), magy_kandil@yahoo.com (Kandil M.), hussein43@hotmail.com (Hussein A. Z.)

\section{To cite this article:}

El-Sayed M. El-Refaie, El-Gamel Amal A., A. H. Gepreel Mohamed, Kandil M., Hussein A. Z. Electrical Properties of Electrorefined Nanostructured Lead $(\mathrm{Pb})$ for Enhancing Radiation Safety. International Journal of Materials Science and Applications.

Vol. 4, No. 5, 2015, pp. 283-287. doi: 10.11648/j.ijmsa.20150405.11

\begin{abstract}
The electrical properties of lead $(\mathrm{Pb})$ nanopowder (metal) produced by "Direct Electrorefining" technique of secondary lead slag are investigated in this study. The morphology and shape of the nanostructure lead powder were examined using a scanning electron microscope (SEM). The particles size is confirmed using a transmission electron microscope (TEM). The good stability of nanoparticles is confirmed during the performed studies. The investigation of the DC conductivity, dielectric constant, dielectric loss and AC conductivity as a function of frequency, temperature of nano-particles are done. The sample's form is a compressed pellets of lead nanoparticles. The results of this study are expected to reflect on understanding the effect of finite nano size powder on the electrical conduction measurements. Plots of the experimental results obtained are reported for various parameters, comparisons are done as well. The successful calculations of temperature dependent resistance value confirm its semi-conducting nature and explore its potential applications in various industries.
\end{abstract}

Keywords: Lead Nanoparticles, Direct Electrofining, DC Conductivity, AC conductivity, Permittivity

\section{Introduction}

Metal nanoparticles are small clusters of metal atoms with size less than $100 \mathrm{~nm}$. [1] Nanomaterials have properties that are different from those of bulk materials. Most nanostructure materials are crystalline in nature, and have unique properties. The properties of nanomaterials are very much different from those at a larger scale. Two principal factors cause the properties of Nano Materials to differ significantly from other materials; (1) Increased relative surface area and (2) Quantum confinement effect. These factors can charge or enhance properties such as reactivity, strength and electrical characteristics. [2] Although metal nanoparticles have been used in various scientific and other fields for some time, their physical and electronic properties are still not fully understood. The potential applications for metal nanoparticles include use in nanoelectronics, electronics with components of nanometer scale. Recently, researchers are trying to reach a detailed understanding of the electrical properties of nanomaterials. In nanocrystals, the electronic energy levels are not continuous as in the bulk but are discrete (finite density of states), because of the confinement of the electronic Wavefunction to the physical dimensions of the particles. This phenomenon is called Quantum confinement. If one length of three dimensional nanostructures is at nano-dimension, then it is called a Quantum Well. If two sides of three dimensional nanostructures are at nano-dimension, then it is called a Quantum Wire. If all three dimensional [4], [5] nanostructures are at nano-dimension (Nano Crystals), they are referred by Quantum Dots (QDs). If the size of a metal cluster is less than the de Broglie wavelength of an electron, conduction electrons will be confined to certain allowed energy levels. The average spacing between allowed energy levels called the Kubo gap will not be apparent at room temperature [5], because the thermal energy is greater than this energy gap. In this case, the nano-particles have metallic conductivity. However, if the thermal energy is less than the Kubo gap, the nano-particle conductivity should be like an insulator [6], [7]. In this study, the electrical properties of metallic lead $(\mathrm{Pb})$ nanopowder, as a crucial material in 
developing nanoelectronics, produced by "Direct Electrorefining" technique of secondary lead slag are investigated [1]. For this $\mathrm{Pb}$ nanopowder, the analysis of the DC conductivity, dielectric constant, dielectric loss and AC conductivity as a function of frequency, temperature of nanoparticles are investigated.

\section{Material and Methods}

\subsection{Material and Sample Preparation}

In this study, the Nano lead powder samples are produced from the recycling of secondary lead slag -comes out from the waste of the industrial production of lead- by electrorefining technique. The direct electrorefining of lead is a new environment friendly technique for metals powder extraction. There are two forms of $\mathrm{Pb}$ powder came out of this recycling process; rods and particles. Hence, this powder was compressed to disc pellets with two thicknesses $0.25 \mathrm{~mm}$ and $0.40 \mathrm{~mm}$ of nano-lead particles and with diameter of $4.95 \mathrm{~mm}$.

The morphology of the lead nanopowder is examined using a scanning electron microscope (SEM) and a transmission electron microscope (TEM).

\subsection{The Current-Voltage (I-V) Characterization of Pb Nano- Powder}

The I-V test was conducted to study the behavior of the $\mathrm{Pb}$ nano-powder compact and to calculate the resistivity of the samples. The basic configuration of the constant voltage method was used. In this method, a constant voltage source, V, is connected in series with the disc pellet sample and the electrometer. Since the voltage drop across an electrometer is negligible, essentially every voltage drop appears is considered across the sample. The resulting current is measured by the electrometer and the resistance is calculated using the Ohm's law. The resistivity is calculated from the geometry of the electrode and the thickness of the sample [8].The currentvoltage characteristics were recorded, plotted the graphed for two samples to assure reliability.

\subsection{Conductivity Measurements}

The DC conductivity of the disc pellet of $\mathrm{Pb}$ nano-powder was measured by holding the sample between two electrodes (made of thick brass blocks) in a conductivity cell using a Keithley (Model 617) electrometer. The test was conducted at room temperature. The basic configuration of a constant voltage method was used. In this method a constant voltage source, $\mathrm{V}$, is connected in series with the pellet sample and with the electrometer. Since the voltage drop across an electrometer is negligible, essentially every voltage drop appears is considered across the sample. The resulting current is measured by the electrometer and the resistance is calculated using the Ohm's laws [9] in equation (1):

$$
\sigma_{D C}=\frac{1}{\rho}=\frac{\mathrm{d}}{\mathrm{AR}}
$$

where $\sigma$ is DC conductivity, $\mathrm{R}$ is the resistance measured, $\rho$ is the resistivity, A is the cross-sectional area of the electrode and $\mathrm{d}$ is the thickness of the sample.

$$
V=\mathrm{IR}
$$

The resistance is the ratio of the potential voltage to the current as in equation (2). It has the unit of ohms $(\Omega)$. The resistivity $(\rho)$ relates the resistance to the volume of the medium (cross-section area A with length L) and is given by equation (1)

\subsection{Dielectric and AC Conductivity Measurements}

The AC conductivity of the disc pellet of $\mathrm{Pb}$ nanoparticles was measured by holdin $g$ the sample between two electrodes. The sample was heated to different temperatures and maintained remained constant within $0.1{ }^{\circ} \mathrm{C}$. The conductivity was also measured (a $25 \mathrm{~V}$ DC was applied across the sample from the constant voltage source provided in the Keithley electrometer). The Dielectric constant, dielectric loss ( $\tan \delta$ ), and AC conductivity of the samples were measured using a Hewlett Packard Model 4192A LF Impedance Analyzer. The dielectric constant $(\varepsilon)$ is one of the basic electrical properties of solids. The measurement of dielectric loss as a function of frequency and temperature-While, the dielectric constant was calculated from the measured capacitance $(C)$ using the relation shown in equation (3)

$$
\varepsilon=\frac{C d}{A \varepsilon_{0}}
$$

where: $d$ is the thickness and $A$ is the area of cross-section of the sample. Then the AC conductivity was calculated using the relation shown in equation (4). The dielectric constant, dielectric loss and AC conductivity measurements were calculated at different frequencies, $f$, (from $50 \mathrm{kHz}$ to $50 \mathrm{MHz}$ ) and over a temperature range (from 25 to $110^{\circ} \mathrm{C}$ ).

$$
\sigma_{a c}=2 \pi f \varepsilon \varepsilon_{0} \tan \delta
$$

\section{Results}

\subsection{Morphology of Pb Nanopowder}

As discussed above, $\mathrm{Pb}$ nanopowders came out of recycling secondary lead slag through the environmental friendly direct electrorefining technique used in this study. The different structures of the powders used in producing pellet sample in this study are represented in figures 1 to 3 .

These figures show some spherical particles among the lead nanorods which indicates that these rods have been made up of spherical particles (depending on the nucleation and growth of particles). It is proposed here that the rods have been formed by binding spherical particles one by one (one over one) and have been elongated in one direction after binding. Due to this, some up and down structures are in the outer surfaces of the nanorods. The surfaces (textures) of the nano-rods are not uniform and not smooth. Aqueous nature of extraction process is probably the reason for the elongation of the nanoparticles in 
one direction and the change of the shape from spherical to rod shape. The synthesized nanoparticles are in various sizes as shown in Fig.3. The particle size range seems varying from $6.84 \mathrm{~nm}$ to $12.95 \mathrm{~nm}$ according to the estimated size measured by TEM analysis only.

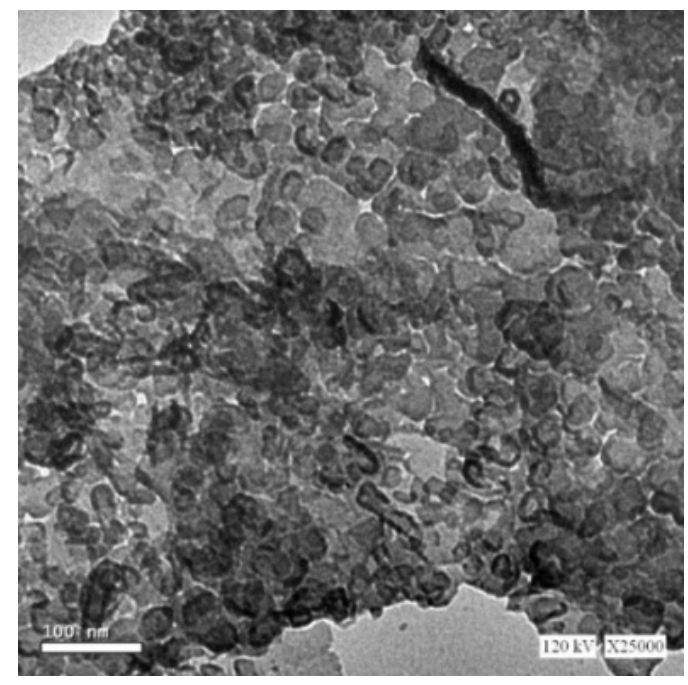

Fig. 1. TEM image of Pb nano-particles.

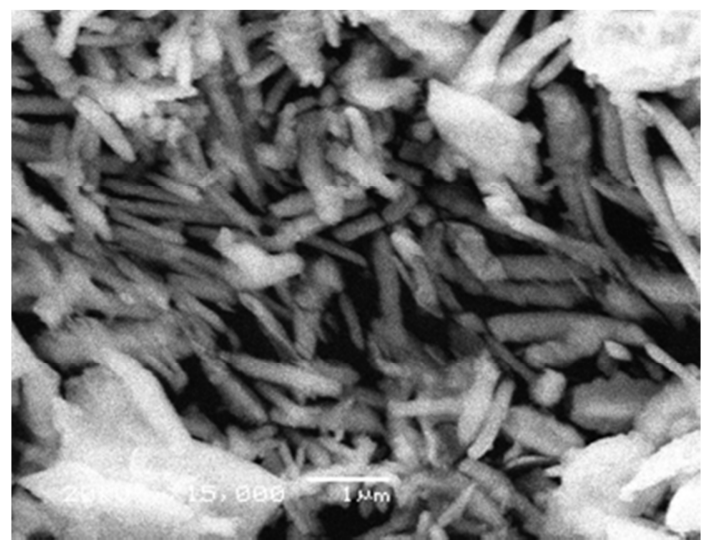

Fig. 2. SEM image of Pb nano-rods.

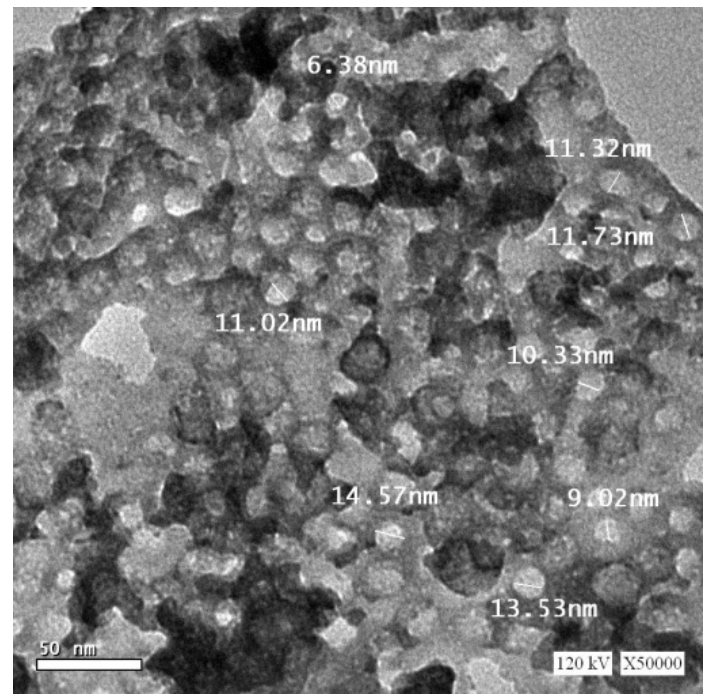

Fig. 3. Higher magnification of TEM pattern of nano particles showing the particle size in the range of $6.38-14.57 \mathrm{~nm}$.

\subsection{I-V Characteristics of Pb Nanopowder}

The Current -voltage characteristics for pellets made of $\mathrm{Pb}$ nanopowders with different thickness samples and conducted at room temperature are measured. The results are shown in figures (4 \& 5).

I. Current-voltage characteristics for nano-structured $\mathrm{Pb}$ pellet of $0.25 \mathrm{~mm}$ thickness was measured as shown in figure.4. The I-V characteristics for a $0.25 \mathrm{~mm}$ thickness disc measured at room temperature can be analyzed by the equations 1 to 4 . The inverse of slope of $\mathrm{I}-\mathrm{V}$ is representing the resistance, $\mathrm{R}=0.109 \times 10^{6} \Omega$ and the resistivity at room temperature, $\rho=0.0084 \times 10^{6} \Omega \cdot \mathrm{m}$, as calculated from equations 1,2 .

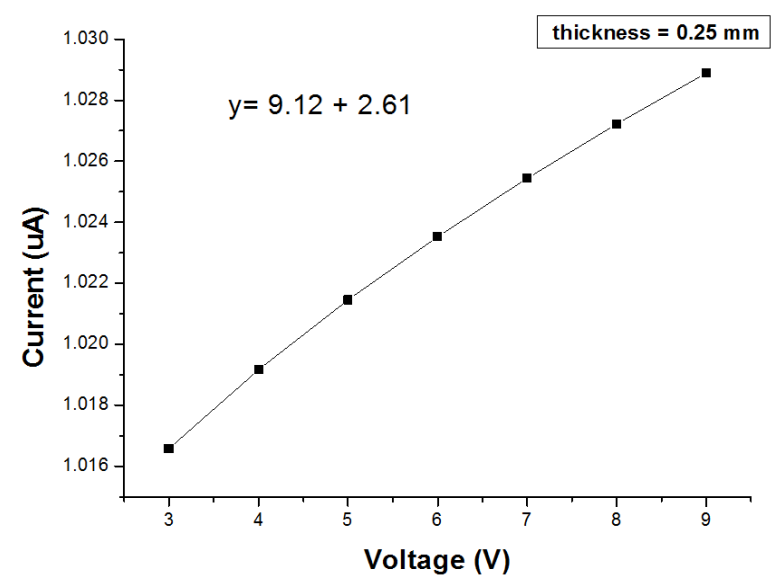

Fig. 4. Current-voltage plot for Pb pellet of $0.25 \mathrm{~mm}$ thickness as measured at room temperature.

II. Current-voltage plot for $\mathrm{Pb}$ pellet of $0.4 \mathrm{~mm}$ thick was measured as shown in figure.5. As observed from Fig5. $\mathrm{Pb}$ pellet of $0.4 \mathrm{~mm}$ thickness showed $\mathrm{R}=3.55 \times 10^{6} \Omega$ and $\rho=$ $0.17 \times 10^{6} \Omega \cdot \mathrm{m}$. It seems that the induced current has been found to increase linearly to some extent due to the change in the applied voltage. In addition, the resistivity decreases with increasing the thickness of the sample although its resistance is increasing.

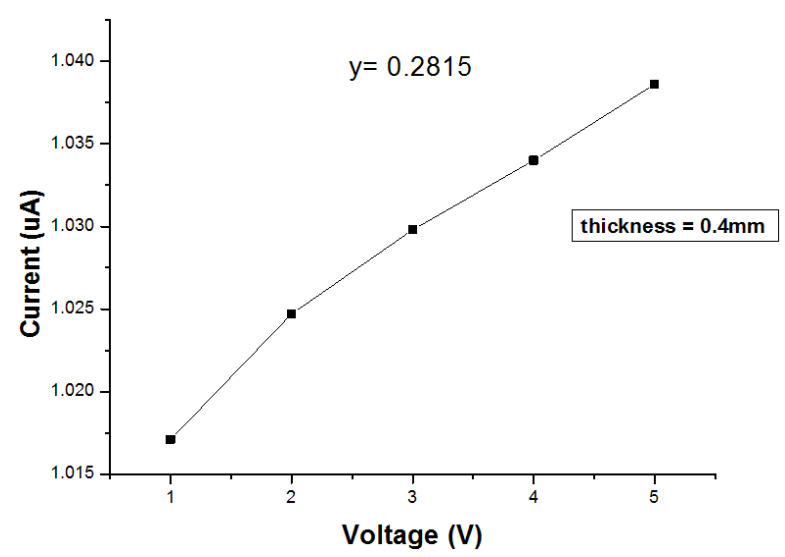

Fig. 5. Current-voltage plot for $P b$ pellet of $0.4 m m$ thickness as measured at room temperature.

In other words, the DC conductivity is decreasing with 
increasing the thickness of the nano-structured $\mathrm{Pb}$ pellet. The compactibility of the $\mathrm{Pb}$ nanopowders into pellet may be a reason for this resistivity difference between different pellet size due to the difference in cavities-contents.

III. Change of resistivity of nano-structured $\mathrm{Pb}$ pellet with temperature. Fig (6) shows that the resistance is decreasing with increasing the temperature, where the current is increasing with increasing the temperature and DC conductivity is increasing with increasing the temperature. This trend is the opposite performance of the bulk metals.

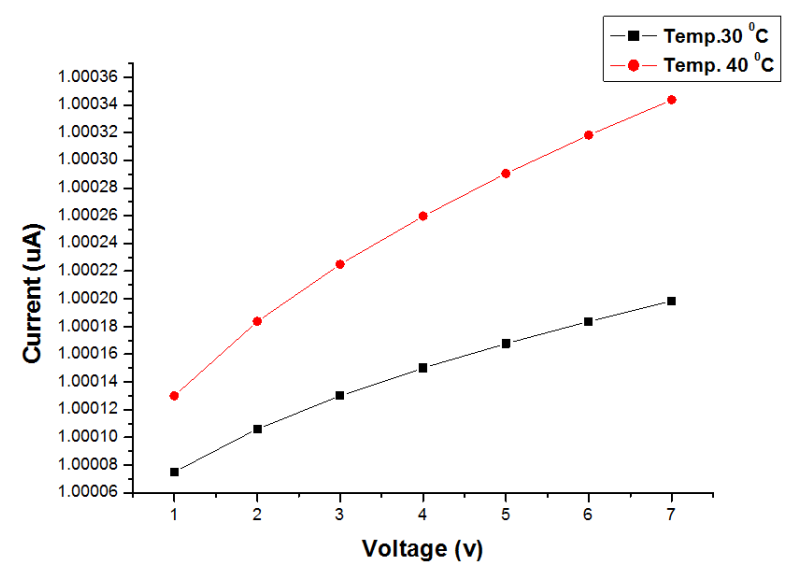

Fig. 6. Plots of current-voltage characteristics that were recorded for a Sample of lead nanoparticlsat different temperature.

$I V$. Fig. 7. Shows plot $\mathrm{I}-\mathrm{V}$ characteristics in different range of frequencies $(50 \mathrm{HZ}-5 \mathrm{MHZ})$ at room temperature. It is observed that, as volt increases, current is increasing at low frequency and constant at the high frequencies. Therefore, DC conductivity increases with temperatures and decreases with the frequencies.

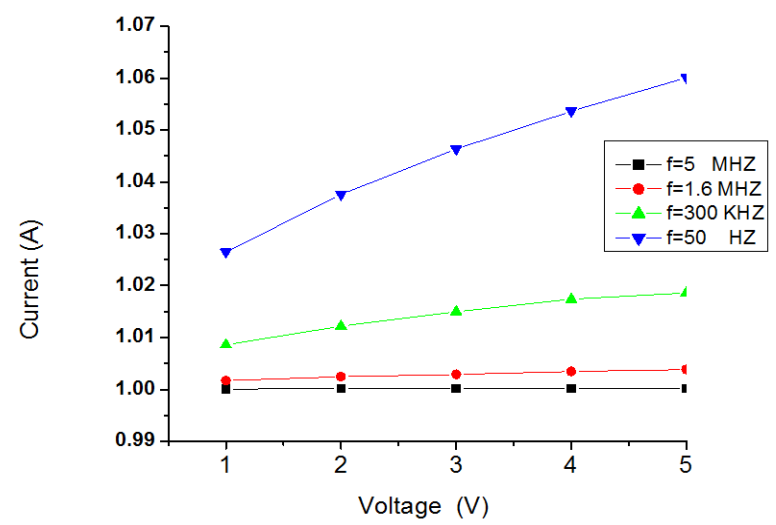

Fig. 7. Plots of current-voltage characteristics of lead nanoparticls at $25^{\circ} \mathrm{C}$ in different frequencies.

$V$. Fig. 8. Shows that the AC conductivity is increasing with increasing temperature and also with increasing frequencies.

The dielectric constant as a function of frequency for the sample is shown in the Fig. 9. It is clear from this figure that it has strong frequency dependence in the lower frequency region. The dielectric constant decreases with the increase of frequency and becomes constant at high frequencies for all temperatures. The dielectric constant assumes constant with the temperature according to this model, a dielectric medium is assumed to be made of well conducting grains which are separated by poorly conducting (or resistive) grain boundaries. Under the application of external electric field, the charge carriers can easily move.

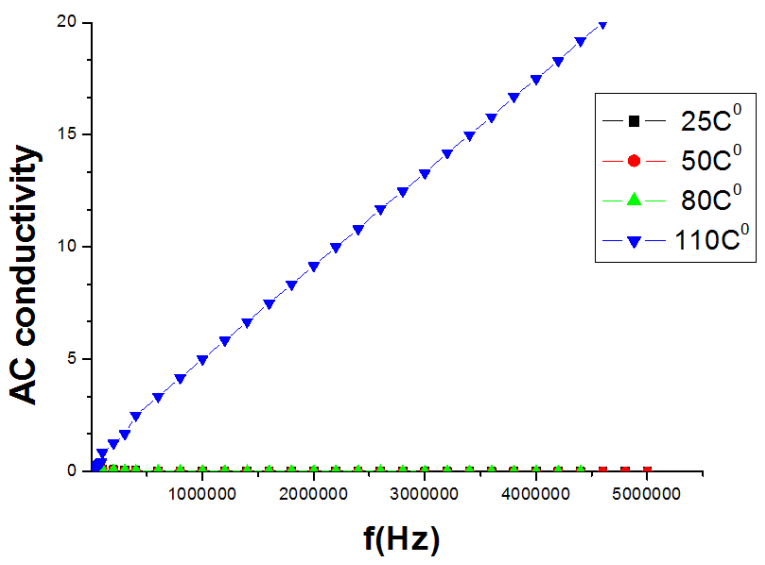

Fig. 8. Variation of AC conductivity with frequency at different temperature.

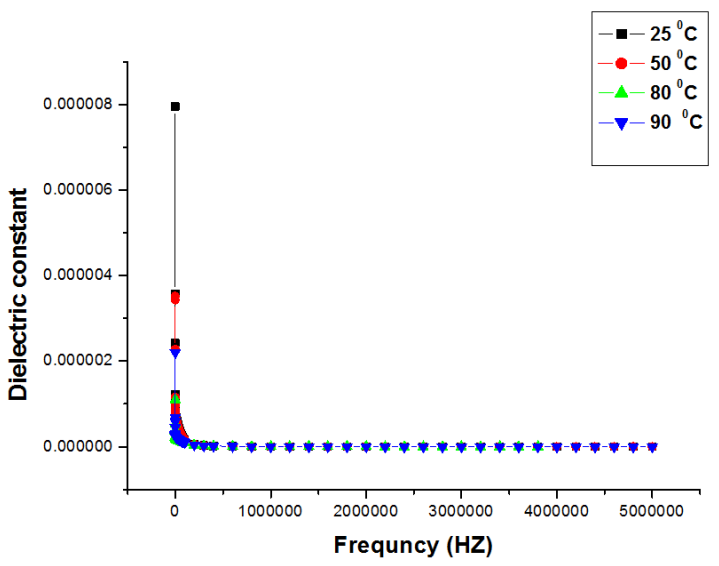

Fig. 9. Variation of dielectric constant with frequency at different temperatures.

VI. It is observed in Fig.10. that the capacitance is inversely proportion with the frequencies but seems constant with temperatures at high frequency and is decreasing slightly with low frequencies.

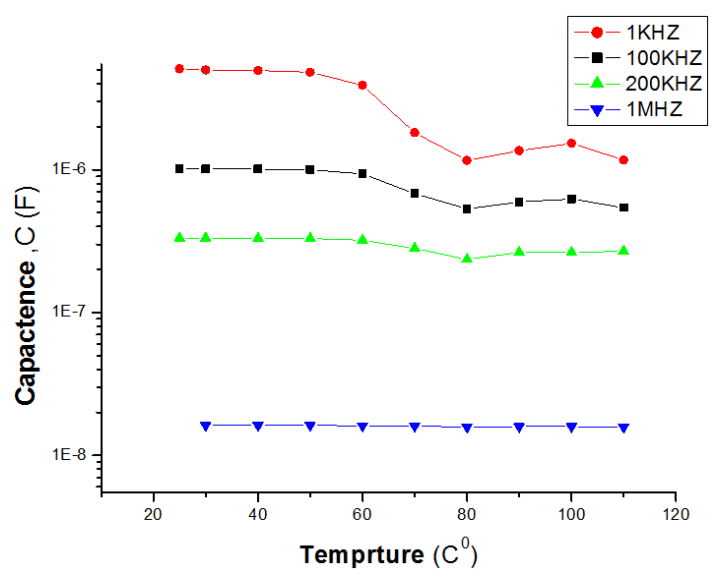

Fig. 10. Variation of capacitance with temperature at different frequencies. 
Loss tangent or loss factor (tan $\delta$ ) represents the energy dissipation in the dielectric system. Fig.11. shows the variation of dielectric loss factor with frequency at different temperatures. It has been observed that $\tan \delta$ decreases at high frequency for all temperatures,-which may be due to the space charge polarization. It can also be seen that $\tan \delta$ increases with increasing the temperatures.

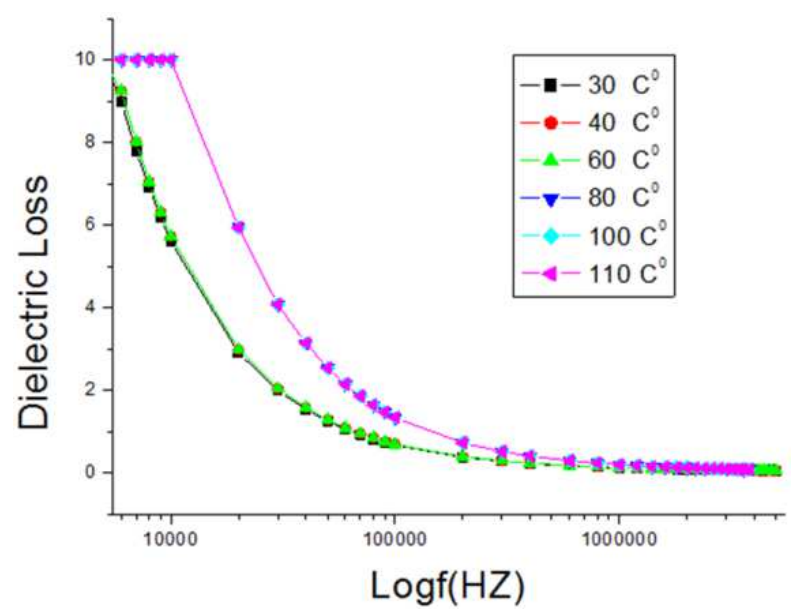

Fig. 11. Variation of dielectric loss with frequency at different temperatures.

\section{Discussion}

In this study, lead nano-particles showed anomalous electro/thermal properties. Generally, metals conductivity decreases when temperature increase because thermal vibrations of crystal lattice disrupt the free motion of electrons. In all metals, although the size of the metal is reduced to the sub-micron scale, resistivity is constant except when they are reduced to nano size scale. For example, gold has a resistivity of $2.44 \times 10^{-8} \Omega \mathrm{m}$ regardless of its size or shape, except when it is nanostructured. Several studies have discussed the explanations of Fuchs' findings [11]. Fuchs and Sondheimer found that the resistivity of thin films increases as the thickness decreases, where the energy levels become discrete rather than continuous. The average spacing between adjacent energy levels in a metal nano-particle is called the Kubo gap, and is related to the Fermi energy of the metal and the size of the nano-particle. For instance, in a lead nano-particle of 3-nm diameter, containing $\sim 10^{3}$ atoms, the Kubo gap is around 5-10 $\mathrm{MeV}$. Therefore, when the thermal energy is greater than this gap at room temperature, the electrical conductivity of nanoparticles will be the same as in bulk metal. But the thermal energy is lowered significantly than the Kubo gap, the nanoparticle becomes an insulator. Here, we have elucidated the importance Kubo gap in governing the emergence of new physicochemical properties of the small metallic particulates. The relation has been found between the metal-insulator transition of metals with variable valence. The criterion of low metallicity (acts as insulator) of lead nano-particles at room temperature and thereafter the conductivity is increasing when temperatures increase, is now understood. Therefore, lead nano-particles act as semiconductor materials at room temperature. Their conductive properties can be modified by controlled addition of impurities or by the application of electrical fields or light.

\section{Conclusion}

It is observed that lead nano-particles have unique electrical properties. Bulk lead metal is a good conductor but lead nanoparticles behave differently as semiconductor at room temperature and its conductivity is increasing with temperature. In addition, its electrical properties are highly affected by the applied frequency. The lead nano-particles is good material for radiation detection.

\section{Acknowledgment}

The authors thank Prof. Ashour Owais of Suez University for producing the materials used in this study.

\section{References}

[1] AshourOwais, Mohamed Abdel-HadyGepreel ;Electrorefining of Secondary Lead Slag Anode Particles, World of Metallurgy - ERZMETALL 66 (5), 5-12

[2] E. Roduner, Nanoscopic Materials: Size-Dependent Phenomena. The Royal Society of Chemistry, Cambridge, 2006.

[3] Yacobi BG. Semiconductor Materials: An Introduction to Basic Principles, Vol.1, Springer, Newyork, 2003. pp. 1-3. ISBN 0306473615.

[4] Youjun He, Guangjin Zhao, Bo Peng, Yongfang Li. HighYield Synthesis and Electrochemical and photovoltaic properties of Indene-C 70 Bisadduct. Adv.Funct.Mater. 2010; 20: 3383-3389. DOI: 10.1002/adfm.201001122.

[5] Theivasanthi T, Alagar M. Konjac biomolecules assisted$\mathrm{rod} / \mathrm{spherical}$ shaped lead nanopowder synthesized by electrolytic process and its characterization Studies. Nano Biomed. Eng. 2013; 5(1): 11-19. doi: 10.5101/nbe.v5i1.p11-19

[6] Bose, S.; Banerjee, R.; Genc, A.; Raychaudhuri, P.;Fraser, H. L.; Ayyub, P. (2006). Size Induced Metal-Insulator Transition in Nanostructured Niobium ThinFilms: Intra-granular and Inter-granular Contributions.J. Phys. Condens. Matter 18: 4553-4566.

[7] Edwards, P. P.; Johnston, R. L.; Rao, C. N. R. (1999). Onthe Size-Induced Metal-Insulator Transition inClusters and Small Particles. In Metal Clusters inChemistry, Vol. 3, ed. Braunstein, P.; Oro, L. A.; P. R.Raithby, Wiley, Weinheim.

[8] W C Michels: Electrical Measurements and their applications, Van Nostrand, New York (1957).

[9] P. Q. Mantas Journal of the European Ceramic Sociecy 19,2079-2086 (1999) "Dielectric response of Materials: Extension to the Debye model".

[10] Bonnie C Baker: "Temperature Sensing technologies" AN 679 Microchip Inc (1 998). SushmaBhat, S K Khosa, P N Kotru, R P Tandon, Muterial Science and Engineering. B 309,7-11 (1995).

[11] Y. Namba, Japanese Journal of Applied Physics 9, 1326 (1969). 